\title{
DEEP LEARNING APPLICATIONS IN MOBILE NETWORKS
}

\author{
Dejan Dašić ${ }^{1,2, *}$, \\ Miljan Vučetić2, \\ Gardelito Hew A Kee ${ }^{3}$, \\ Miloš Stanković1, 2
}

\author{
'Singidunum University, \\ Belgrade, Serbia \\ 2 Vlatacom Institute, \\ Belgrade, Serbia \\ ${ }^{3}$ European University, \\ Belgrade, Serbia
}

Correspondence:

Dejan Dašić

e-mail:

dejan.dasic@vlatacom.com

\begin{abstract}
:
The expected deployment of 5G networks, growing percentages of mobile networks' market penetration levels and increasing popularity of mobile applications induce new challenges and requirements for mobile network operators. One of the ways of tackling these new challenges is the deployment of advanced machine learning techniques in hopes of handling expected traffic volumes, performing real-time analytics and managing network resources. The paper presents the aspects of mobile networking in which deep learning methods can be implemented. After presenting the basic background and modern deep learning techniques and related technologies, paper presents an overview of the current advances made in these research areas. The paper also identifies the fields within the realm of mobile networking that show particular potential for new exploration.
\end{abstract}

\section{Keywords:}

Deep learning, mobile networks, mobile data analysis, network security, signal processing.

\section{INTRODUCTION}

Modern day and upcoming mobile networks generate immense volumes of various heterogeneous data. Implementation of deep learning structures and algorithms, as opposed to traditional machine learning (ML), is advised as the performance of deep learning increases significantly with the growth of size of training data sets. Furthermore, training deep NNs with big data sets prevents model over-fitting. On the other hand, application of Stochastic Gradient Descent (SGD) training method to NNs requires only sub-sets of data at each training step, guarantying scalability of deep learning with big data [1].

Data generated in mobile networks is heterogeneous and noisy in nature and exhibits complex spatial and temporal patterns [2]. These features only amplify another advantage of deep learning over traditional ML algorithms, which is the ability of extraction of high-level features from the data of complex structure and with inner correlations. Labeling of such data would otherwise require significant human effort, which is costly [3]. 
Another advantage of implementation of deep learning over traditional machine learning in the area of mobile networking comes from the fact that deep learning provides several methods which enable use of unlabeled data for the purposes of extraction of useful patterns through unsupervised learning. This is important as most current mobile systems generate semi-labeled or unlabeled data [2].

In the following section (section II) the paper introduces the basics of deep learning, giving the explanation of the neural network (NN) and briefly presenting the deep learning architectures referenced in the sections to follow. In section III we name the areas within mobile networking in which implementations of deep learning have been investigated by the researchers. A brief overview of the advances in these application areas is also presented. Section IV concludes the paper, summarizes some of the challenges and sheds light on future research directions.

\section{DEEP LEARNING BASICS}

Deep Learning is a subfield of Machine Learning dealing with structures and algorithms meant to mimic the operation of the human brain. The best-known model to serve as the basis for deep learning is an artificial neural network. Neural network, imitating the brain structure, is comprised of layers of neurons, interconnected by weighted connections. For the purposes of modeling such structures the neurons can be considered as variables and the weights assigned to connections between them can be regarded as parameters. The purpose of network training is to configure the network parameters is such a way so as to ensure that the desired set of inputs causes the desired set of outputs [4]. The term "deep" neural network refers only to neural networks with sufficient number of hidden layers. Implementation of deep learning models enables making predictions, classifications or decisions based on the input set of data without explicitly programming, but rather learning the network. Other structures, beside deep neural networks, comprising of multiple layers, such as deep random forests [5] or deep Gaussian processes [6] can also be considered deep learning structures.

One of the structures widely used in deep learning applications is the Multilayer Perceptron (MLP). It represents a network of several layers of operations whose outputs depend on the inputs applied with weights and biases [7]. Being the original artificial neural network it was also the most popular one in the past. But due to its fully-connected units between layers contributing to its complexity, this architecture is growing less popular. It is, however, used in more complex structures like Convolutional Neural Networks (CNNs).

Unlike MLPs that employ full set of connections between the layers, CNNs use a set of locally connected filters in order to extract correlations between data regions. This gives CNNs extraordinary performance in imaging applications[8].

Another widely used architecture in machine learning is a Restricted Boltzmann Machine (RBM). RBMs are structures made of two layers - a, so called, visible and a hidden layer - in which units can take binary values with certain probabilities. Hidden units are fully connected to visible ones with weights, but unlike in MLPs where the hidden layers are affected by the inputs (visible layer) - here the hidden layer affects the visible layer as well. Several RBMs stacked together form a structure called a Deep Belief Network (DBN) [9].

Auto-Encoders (AE) are unsupervised learning structures that attempt to copy their inputs to their outputs over several hidden layers between them, while performing dimensionality reduction in the process [10].

Recurrent Neural Networks (RNN) is a structure whose current state depends on the inputs as well as the previous states of the network. As such, these networks are well suited for modelling sequential data [11].

An idea of remembering the state of the network in order to use it in the future is maybe best implemented in Long Short-Term Memory (LSTM) networks in which each unit has three gates (input, output, forget) by which to control the information flow to itself [12].

Generative Adversarial Network (GAN) is a framework in which two models, i.e. neural networks, are trained simultaneously. One model is a generative one and it tries to approximate the target data distribution from the training set data, whereas the other model is a discriminative one trying to guess if the sample at its input comes from the real training data or the output of a "counter-part" generative network [13].

All of the structures named previously can be trained using one or more of the three machine learning training approaches: unsupervised learning, supervised learning and reinforcement learning.

Supervised learning is the teaching method in which the network is fed with labeled data pairs (input-output) in order to produce a desired output for a given input. Unsupervised learning is a learning method without the labeled data in which the network is expected to perform 
some classification of the input data based on the existence of commonalities within the data samples. Reinforcement learning is a paradigm which introduces an idea of a reward, whereas the actions of certain agent influence the environment to a new state and bring about the reward for that action. The reward and the new state form basis for a new action of the agent. Reinforcement learning is an attempt to approximate the environment so as to maximize the rewards [14] .

\section{DEEP LEARNING APPLICATIONS IN MOBILE NETWORKS}

In the following subsections we describe the areas of mobile networking in which deep learning has made significant advances:

\section{Mobile Data Analysis}

Sources of data within the mobile network include infrastructure elements' metadata, performance indicators, call records, radio information, end user devices, user profiles, applications and sensors on end devices. This makes mobile networks a true big data source with the data storage being partly cloud-based and partly edge-based. Authors in [15] categorize mobile network data into a group of network-level data and applicationlevel data based on the data source localization.

\section{Network-level data}

Part of the reason for spatial and temporal variations in network-level data is users' varying behavior [16]. This fact makes this data good basis for user mobility analysis and public transportation planning [17], as well as network diagnosis and management.

Part of the efforts employing network-level data goes to the aim of network state prediction. These activities relate to prediction of network traffic or performance indicators while considering historical measurements or related data. Authors in [18] demonstrate high accuracy of prediction of Quality of Experience (QoE) by employing MLPs and using objective metrics such as average user throughput, number of active users in a cell, average data volume per user, and channel quality indicators as their inputs. Part of the efforts goes into implementation of deep learning with the idea of city-scale mobile traffic forecasting, by using spatio-temporal correlations of geographic mobile traffic measurements. A time sequence of mobile traffic data measurements taken over a region is used to predict mobile traffic in future time instances. Authors in [19] implement AEs for extraction of spatial feature and dimension reduction and then process the extracted representations by LSTMs in order to perform the final forecasting. This arrangement applied to real-world datasets shows performance superior to Support-Vector Machines (SVM) and the Autoregressive Integrated Moving Average (ARIMA) model. Some authors ([20], [21], [22] and [23]) employ deep learning to CNNs and LSTMs for the purpose of mobile traffic forecasting. They also show accuracy higher than traditional methods such as ARIMA. Some authors inspired by techniques of image super resolution [17] investigate the possibility of employing dedicated CNNs in combination with GAN in order to predict with fine resolution the network-wide mobile traffic consumption by using coarse-grained measurements. This is done in hopes of reducing measurement overhead. Read-world dataset experiments show improvement in measurement granularity of up to 100 times.

Another area of research interest of using networklevel data in deep learning implementation is mobile traffic classification. Deep AEs have been used by authors in [24] to identify protocols in TCP flow datasets with great precision. For the purposes of encrypted traffic classification authors in [25] used MLPs, CNNs and LSTMs. For the same purpose authors in [26] propose a $1 \mathrm{D} \mathrm{CNN}$ which seems to be a promising architecture due to its reduced complexity. CNN structures are also used for the purpose of identification of malware traffic in [27] where mobile data are regarded as images. Representation learning is then used to classify unusual patterns which represent malware traffic.

Part of the network-level data resides in the Call Data Register (CDR) and mining of CDR data can provide useful information. As RNNs are well suited for sequential data analysis, in [28] user trajectories from streaming CDR data are considered as sequence of locations and RNNs are used to estimate metro density. CDR data are also used in [29] where CNNs are employed for the purposes of users' gender and age predictions.

\section{Application-level data}

Application-level data is sourced from various mobile devices. The devices act as local sensor hubs used for 
data collection and preprocessing [30]. This heterogeneous data, from sources such as GPS sensors, accelerometers, mobile cameras and video recorders, even portable medical monitors can be collected through crowd-sourcing schemes for further analysis. This data is highly related to user's profile and thus reflects users' behavior, such as mobility, preferences and personal communication patterns [31]. Extraction of this data can be used in recommender systems and user targeted advertising. Some of this data is private. Extraction of useful patterns from end-user multi-modal sensing devices without raising privacy issues is still a challenge.

Two approaches are implemented in applicationlevel mobile data analysis. One is cloud-based computing and the other is edge-based computing; difference between the two being the localization of the actual computing object. In cloud-based computing the inference is done in a cloud and the results are sent to edge devices, whereas in edge-based computing models are deployed on edge devices themselves which perform local calculations.

One popular use of application-level data is in the area of mobile health where wearable sensors capture physical condition data of or around their users. In one such application sensory devices are used to warn disabled persons (deaf) of the various recognized emergency situations [32]. In other works deep learning networks are used to classify lifestyle of users based on wearable devices sensory information or to perform edge-computing health monitoring.

Significant interest and use of application-level mobile data is seen in the area of pattern recognition, be it for object classification or activity recognition. Some research is this area [33] investigates the possibility of employing CNNs on mobile devices for the purpose of object recognition. Authors compare task performance on CPU, GPU and mobile platforms and show the possibility of successful implementation of such platform on a mobile device. Efforts in the field of activity recognition range from recognition of human activity by analysis of offline smart phone datasets gathered by accelerometers and gyroscopes [34] or the analysis of sensory data collected by a smart watch by using RBM [35] to designing a virtual "X-ray" machine to recognize human poses based on radio frequency signals analyzed by a proposed 4D CNN framework [36].

Application-level mobile data is also used in the area of Automatic Speech Recognition (ASR). Work in [37] presents a specific architecture in a CNN model which facilitates ASR using mobile multi-microphone devices in noisy environments. In [38] authors propose and evaluate a new deep learning framework for the purpose of multi-task audio sensing. They test on a smartphone performing the tasks of speaker identification, emotion recognition, stress detection, and ambient scene analysis and show great accuracy while achieving high runtime, memory and energy efficiency.

\section{Mobility Analysis}

Movement patterns of individuals groups that can be extracted from mobile network data can be a highly useful resource in the processes of urban planning or public service provisioning, or network resource management. Due to its ability to extract spatial dependencies in sequential data deep learning is well suited for mobility analysis. Authors in [39] employ LSTM networks to model the joint movement patterns of a large group of people and vehicles city-wide. In [40] authors use RNNs to perform short-term mobility forecasting on a realworld deployment dataset and show accuracy superior to traditional, non-deep approaches.

\section{User Localization}

Location based applications and services in mobile network environment require knowledge of individual user positioning. Some work of implementing deep learning to this end is presented in [41] where the authors use crowd-sensed geo-tagged received signal strength information to learn an MLP of the correlation between the cellular signals and the location of the users.

\section{Network Control}

Advances made by deep learning, based on its powerful function approximation mechanism, in improving traditional reinforcement learning and imitation learning gave rise to efforts of tackling network control problem known for their complexity. Besides reinforcement learning and imitation learning the approach implemented in network control is analysis-based control. Analysis-based control does not output actions direct$l y$, as do the reinforcement and imitation learning approaches, but rather extracts the useful information and provides it to an agent for further action.

One form of network control is network optimization which means management of network resources in 
order to improve network performance. Utilization of mobile data analytics enables prediction of spatial and temporal changes in the requirements for network resources [31]. This means that the network operators can allocate radio resources in the manner required so as to optimally fulfil the user demand. Authors in [42] use LSTMs to predict traffic load at base station in ultradense networks. Based on these predictions they change resource allocation policy so as to avoid congestion. Authors in [43] make use of the RNN for traffic prediction with the idea to help dynamic spectrum assignment in a mobile network. Some research investigates network operation under adversarial circumstances. Authors in [43-14] address the issue of anti-jamming communication in unknown and dynamic environments with a deep reinforcement learning agent. They combine deep Q-network with CNN and take raw spectrum information as the input. With limited prior knowledge of the environment they improve the network throughput.

\section{Network Security}

Network security deals with the protection of network resources and entities from unauthorized access, information leakage and malicious attacks. This function is achieved by implementation of firewalls, anti-virus software and intrusion detection systems. Modern day cyber security systems implement deep learning both in the forms of supervised and unsupervised learning. Application of supervised learning enables recognition of undesired signatures and patterns from previous experience and their generalization applied to future intrusions. Application of unsupervised learning enables identification of patterns clearly different from normal behavior. One thing of importance to consider is that besides the protection perspective, deep learning can also be used from the attack perspective.

On the infrastructure level research goes in the direction of identification of network events that fall out of expected behavior, thus indicating a possible attack. Stacked AE is used to categorize network traffic with high accuracy [44] or in combination with MLP for feature selection and extraction [45]. AEs are also used in [46] to detect abnormal spectrum usage.

Security on a software level addresses mainly the issues of smartphone malware and botnets in modern mobile networks. Deep learning is implemented for analysis and detection of these threats.

RBMs are used in [47] to classify Android malware by training on both labeled and unlabeled mobile applications. Other approaches to tackling the malware detection go the way of analysis of essential application features, where the malware features are extracted by DBNs and SVMs are used for classification. CNNs are also used in malware detection. In [48] detection of malware is done by employing CNNs to detect sequences of opcodes indicative of malware from a disassembled byte-code of an application used as text for analysis.

MLPs or LSTMs can be used for the purpose of detection of botnets through feature extraction from mobile botnet behaviors.

Security implementations of deep learning on a level of user privacy are in good part concerned with preservation of user private data in the process training and evaluating a deep neural network. In several studies researchers implement a deep learning framework partitioned across the cloud and mobile devices, where the feature extraction actions are performed on the mobile devices and classification is performed in the cloud. Local processing of input data ensures strong privacy guarantees.

Another implementation of deep learning networks in research has been for performing cyber-attacks with the purpose of compromising private user information or predicting, i.e. guessing passwords. GAN architectures are mostly used for these purposes. Various deep learning architectures are used in [49] in order to perform side channel key recovery attacks. Compared to other template machine learning attacks, deep learning based methods show better performance in breaking unprotected or protected implementations of Advanced Encryption Standard. Research in [50] shows that CNNs can guess with high accuracy the list of active applications run on a smartphone using only data on orientation and data from a magnetometer. Should data from motion sensors be added to the list of used data, the accuracy levels would reach $98 \%$ which could pose a threat to user privacy. Research also shows that injection of Gaussian noise to the used data significantly reduces inference accuracy, thus mitigating the threat to user privacy.

\section{Signal Processing}

Deep learning techniques are finding their ground in signal processing application, mostly in the areas of Multiple-Input-Multiple-Output (MIMO) processing and modulation.

Deep learning helps optimize MIMO performance based on environmental conditions. MLP based networks 
are investigated as possible estimators of transmitted vectors in a MIMO channel in [51] where simulations show that this architecture achieves high accuracy with light computation requirement.

For the purposes of channel estimation and signal detection researchers use CNNs as well as MLPs.

Researchers in [52] suggest implementation of noniterative neural networks for the purpose of control of transmit power of base stations with the aim of preventing inter-cell interference. Neural network estimates the optimal transmit power at every packet transmission. Simulations show performance better than that of a currently used belief propagation algorithm.

Researchers in [53] bring deep learning to physical layer design. Authors observe and treat a single-user end-to-end MIMO system as an unsupervised deep AE in which MLP followed by a normalization layer forms the transmitter. The receiver is formed of another MLP and a Gaussian noise layer acts as the channel. Experiments show that such an AE system performs considerably better than the Space Time Block Code approach.

Tests of deep learning platforms regarding modulation recognition show that LSTMs are the best candidates for performing this task. However, it has been proven that CNNs can also provide satisfying accuracy in modulation classification.

\section{CONCLUSION}

In this paper, current and future applications of deep learning techniques to mobile networking have been discussed. Advantages of using these techniques in several areas of mobile networks have been stressed.

Certain drawbacks of deep learning do, however, restrict its applicability in mobile networking. Deep learning is, generally speaking, vulnerable to adversarial examples. It is also known low interpretability and requires massive volumes of data to training.

One direction of future efforts in the mobile networking applications of deep learning can be expected to be creation of massive high-quality datasets for the purposes of networks' training. Because of the rising importance of location based services, advances in the field of application of deep learning for the purposes of spatio-temporal data mining can also be expected [54]. Due to large amounts of unlabeled data created by the networks, future research efforts can also be expected in the field of unsupervised learning with the goal of labeling and classifying said data. For the purposes of network control, developments in the field of (deep) reinforcement learning applications are foreseen.

\section{REFERENCES}

[1] C. Zhang, P. Patras, H. Haddadi, "Deep Learning in Mobile and Wireless Networking: A Survey", IEEE Communications Surveys \& Tutorials, March 2019.

[2] M.A. Alsheikh, D. Niyato, S. Lin, H.P. Tan, and Z. Han, "Mobile big data analytics using deep learning and Apache Spark". IEEE network, 2016, 30(3):22-29.

[3] P. Domingos, "A few useful things to know about machine learning", Communications of the ACM, 2012, 55(10):78-87.

[4] C. Jiang et al., "Machine Learning Paradigms for Next-Generation Wireless Networks IEEE Wireless Communications", April 2017, 24(2): 98-105.

[5] M. Garnelo et al. "Neural processes", unpublished

[6] A. Damianou, N. Lawrence, "Deep Gaussian processes”, Artificial Intelligence and Statistics, 2013, pp. 207-215.

[7] R. Collobert, S. Bengio, "Links between perceptrons, MLPs and SVMs", Proc. twenty-first ACM international conference on Machine learning, 2004, p. 23.

[8] A. Krizhevsky, I. Sutskever, G. Hinton, "Imagenet classification with deep convolutional neural networks", Advances in neural information processing systems, 2012, pp. 1097-1105

[9] G. Hinton, S. Osindero, Y.W. The, "A fast learning algorithm for deep belief nets", Neural computation, 2006, 18(7):1527-1554.

[10] Y. Bengio et al., "Learning deep architectures for AI", Foundations and trends in Machine Learning, 2009, 2(1):1-127.

[11] I. Sutskever, O. Vinyals, Q. Le, "Sequence to sequence learning with neural networks", Advances in neural information processing systems, 2014, pp. 3104-3112.

[12] F. Gers, J. Schmidhuber, F. Cummins, "Learning to forget: Continual prediction with LSTM", 1999.

[13] M. Arjovsky, S. Chintala, L. Bottou, "Wasserstein generative adversarial networks", Proc. International Conference on Machine Learning, 2017, pp. 214-223.

[14] X. Liu, Y. Xu, L. Jia, Q. Wu, A. Anpalagan, “Anti-jamming Communications Using Spectrum Waterfall: A Deep Reinforcement Learning Approach", IEEE Communications Letters, 2018, 22(5):998-1001.

[15] D.Z. Yazti, S. Krishnaswamy. "Mobile big data analytics: research, practice, and opportunities", Proc. 15th IEEE International Conference on Mobile Data Management (MDM), 2004, volume 1, pages 1-2 
[16] D. Naboulsi, M. Fiore, S. Ribot, R. Stanica, "Largescale mobile traffic analysis: a survey", IEEE Communications Surveys \& Tutorials, 2016, 18(1):124161.

[17] C. Zhang, X. Ouyang, P. Patras, "ZipNet-GAN: Inferring fine-grained mobile traffic patterns via a generative adversarial neural network", Proc. 13th ACM Conference on Emerging Networking Experiments and Technologies, 2017.

[18] L. Pierucci and D. Micheli, "A neural network for quality of experience estimation in mobile communications", IEEE MultiMedia, 2016, 23(4):42-49.

[19] J. Wang et al., "Spatiotemporal modeling and prediction in cellular networks: A big data enabled deep learning approach", Proc. 36th Annual IEEE International Conference on Computer Communications (INFOCOM), 2017.

[20] C.W. Huang, C.T. Chiang and Q. Li, "A study of deep learning networks on mobile traffic forecasting", Proc. 28th IEEE Annual International Symposium on Personal, Indoor, and Mobile Radio Communications (PIMRC), 2017, pp. 1-6.

[21] J. Feng, X. Chen, R. Gao, M. Zeng, Y. Li, “DeepTP: An End-to-End Neural Network for Mobile Cellular Traffic Prediction", IEEE Network, 2018, 32(6):108-115.

[22] I. Alawe, A. Ksentini, Y. Hadjadj-Aoul, P. Bertin, "Improving traffic forecasting for $5 \mathrm{G}$ core network scalability: A Machine Learning approach", IEEE Network, 2018, 32(6):42-49.

[23] L. Chen et al., "Deep mobile traffic forecast and complementary base station clustering for C-RAN optimization", Journal of Network and Computer Applications, 2018, 121:59-69.

[24] Z. Wang, "The applications of deep learning on traffic identification", BlackHat USA, 2015.

[25] G. Aceto, D. Ciuonzo, A. Montieri, A. Pescapé, "Mobile encrypted traffic classification using deep learning", Proc. 2nd IEEE Network Traffic Measurement and Analysis Conference, 2018.

[26] W. Wang, M. Zhu, J. Wang, X. Zeng, Z. Yang, "Endto-end encrypted traffic classification with onedimensional convolution neural networks", Proc. IEEE International Conference on Intelligence and Security Informatics, 2017.

[27] W. Wang, M. Zhu, X Zeng, X.Ye, Y. Sheng, "Malware traffic classification using convolutional neural network for representation learning", Proc. IEEE International Conference on Information Networking (ICOIN), 2017, pp. 712-717.

[28] V. Liang et al.,"Mercury: Metro density prediction with recurrent neural network on streaming CDR data", Proc. IEEE 32nd International Conference on Data Engineering (ICDE), 2016., pp. 1374-1377.
[29] B. Felbo, P.Sundsøy, A. Pentland, S. Lehmann, Y.A. de Montjoye, "Using deep learning to predict demographics from mobile phone metadata",Proc. workshop track of the International Conference on Learning Representations (ICLR), 2016.

[30] X. Cheng, L. Fang, X. Hong, L. Yang, "Exploiting mobile big data: Sources, features, and applications", IEEE Network, 2017, 31(1):72-79.

[31] K. Zheng et al., "Big data-driven optimization for mobile networks toward 5G", IEEE Network, 2016, 30(1): 44-51.

[32] S. Liu, J. Du, "Poster: Mobiear-building an environmentindependent acoustic sensing platform for the deaf using deep learning", Proc. 14th ACM Annual International Conference on Mobile Systems, Applications, and Services Companion, 2016, pp. 50-50.

[33] L. Tobías, A. Ducournau, F. Rousseau, G. Mercier, R. Fablet, "Convolutional neural networks for object recognition on mobile devices: A case study", Proc. 23rd IEEE International Conference on Pattern Recognition (ICPR), 2016, pp. 3530-3535.

[34] B. Almaslukh, J. AlMuhtadi, A. Artoli, "An effective deep autoencoder approach for online smartphonebased human activity recognition", International Journal of Computer Science and Network Security (IJCSNS), 2017, 17(4):160.

[35] S. Bhattacharya, N. Lane, "From smart to deep: Robust activity recognition on smartwatches using deep learning", Proc. IEEE International Conference on Pervasive Computing and Communication Workshops (PerCom Workshops), 2016, pp. 1-6.

[36] M. Zhao et al., "RF-based 3D skeletons", Proc. ACM Conference of the ACM Special Interest Group on Data Communication (SIGCOMM), 2018, pp. 267-281.

[37] T. Yoshioka et al.,"The NTT CHiME-3 system: Advances in speech enhancement and recognition for mobile multi-microphone devices", Proc. IEEE Workshop on Automatic Speech Recognition and Understanding (ASRU), 2015, pp. 436-443.

[38] P. Georgiev, S. Bhattacharya, N. Lane, C.Mascolo, "Low-resource multi-task audio sensing for mobile and embedded devices via shared deep neural network representations", Proc. ACM on Interactive, Mobile, Wearable and Ubiquitous Technologies (IMWUT), 2017, 1(3):50.

[39] X. Song, H. Kanasugi, R. Shibasaki, "DeepTransport: Prediction and simulation of human mobility and transportation mode at a citywide level", Proc. International Joint Conference on Artificial Intelligence, 2016, pp. 2618-2624.

[40] R. Jiang et al., "DeepUrbanMomentum: An Online Deep-Learning System for Short-Term Urban Mobility Prediction", Proc. National Conference on Artificial Intelligence (AAAI), 2018. 
[41] A. Shokry, M. Torki, M. Youssef, "DeepLoc: A ubiquitous accurate and low-overhead outdoor cellular localization system", Proc. 26th ACM SIGSPATIAL International Conference on Advances in Geographic Information Systems, 2018, pp. 339-348.

[42] Y. Zhou, Z.M. Fadlullah, B. Mao, N. Kato, "A DeepLearning-Based Radio Resource Assignment Technique for 5G Ultra Dense Networks", IEEE Network, 2018, 32(6):28-34.

[43] H. Rutagemwa, A. Ghasemi, S. Liu, "Dynamic spectrum assignment for land mobile radio with deep recurrent neural networks", Proc. IEEE International Conference on Communications Workshops (ICC Workshops), 2018.

[44] V.L.L. Thing, "IEEE 802.11 network anomaly detection and attack classification: A deep learning approach", Proc. IEEE Wireless Communications and Networking Conference (WCNC), 2017, pp. 1-6.

[45] M.E. Aminanto, K. Kim, "Detecting impersonation attack in WiFi networks using deep learning approach", Proc. International Workshop on Information Security Applications, Springer, 2016, pp. 136-147.

[46] Q. Feng, Z. Dou, C. Li, G. Si, “Anomaly detection of spectrum in wireless communication via deep autoencoder", Proc. International Conference on Computer Science and its Applications, Springer, 2016, pp. 259-265.

[47] Z. Yuan, Y. Lu, Z. Wang, Y. Xue, "DroidSec: deep learning in Android malware detection", ACM SIGCOMM Computer Communication Review, 2014, 44:371-372.
[48] N. McLaughlin et al., "Deep android malware detection", Proc. Seventh ACM on Conference on Data and Application Security and Privacy, 2017, pp. 301-308.

[49] H. Maghrebi, T. Portigliatti, E. Prouff, "Breaking cryptographic implementations using deep learning techniques", Proc. International Conference on Security, Privacy, and Applied Cryptography Engineering, Springer, 2016, pp. 3-26.

[50] R. Ning, C. Wang, C. Xin, J. Li, H. Wu, “Deepmag: sniffing mobile apps in magnetic field through deep convolutional neural networks", Proc. IEEE International Conference on Pervasive Computing and Communications (PerCom), 2018, pp. 1-10.

[51] N. Samuel, T. Diskin, A. Wiesel, "Deep MIMO detection", arXiv preprint arXiv:1706.01151, 2017.

[52] M.A. Wijaya, K. Fukawa, H. Suzuki, "Intercell-interference cancellation and neural network transmit power optimization for MIMO channels", Proc. IEEE 82nd Vehicular Technology Conference (VTC Fall), 2015, pp. 1-5.

[53] T.J. O'Shea, T. Erpek, T.C. Clancy, "Deep learning based MIMO communications", arXiv preprint arXiv:1707.07980, 2017.

[54] A. Fumo, M. Fiore, R. Stanica, "Joint spatial and temporal classification of mobile traffic demands", Proc. IEEE Conference on Computer Communications, 2017, pp. 1-9. 